\title{
Strategies to Improve Outcome and Value of the Immune Check Point Inhibitors in Non-Small-Cell Lung Cancer
}

\author{
Helmy M. Guirgis ${ }^{1 *}$ \\ 'The Cancer Free Foundation, 24806 Sea Crest Dr. Dana Point, CA 92629
}

Article Info

Article Notes

Received: April 10, 2018

Accepted: April 26, 2018

\section{*Correspondence:}

Dr. Helmy M. Guirgis, The Cancer Free Foundation, 24806 Sea Crest Dr. Dana Point, CA 92629, USA;

Email: cancerguir@gmail.com

C 2018 Guirgis HM. This article is distributed under the terms of the Creative Commons Attribution 4.0 International License.
Over the last 10 years, there has been a steady progress in the development of anticancer drugs in non-small cell-lung cancer (NSCLC). Progress, however, has come at high costs. Rising drug costs and stagnant or diminishing value are of major concern to governments, drug authorities and patients all over the globe. In the United States (US), the average 4-week costs of new anticancer drugs are estimated at $\$ 10,000$. We recently reported on the impact of PD-L1 on survival and value of the immune check point inhibitors (ICPI) (1). The present communication is a review of our findings. The objectives were to weigh drug costs vs. value and facilitate full disclosure and transparency of outcome and costs to patients. The methodology is briefly summarized as:

1-A scale was developed to grade overall survival gain over control (OS). Grade D was assigned to $<60$ days in view of the growing recognition among the oncology community that OS gains of $<2$ months might be clinically insignificant.

2 -Value was expressed relative to $\$ 100,000$, the average acceptable cost-effectiveness ratio (ACER) in the US. The simplicity and rapidity of the methodology would render the 2 platforms as screening tools for value by the busy community oncologists, pharmacists and nurses. Calculations were carried out in few minutes once the data were entered.

The results demonstrated that in 2nd-line NSCLC, the 3-ICPI analysed namely Nivolumab (Nivo), Atazolizumab (Atezo) and Pembrolizumab (Pembro) did not carry the black box warnings assigned by the Federal Drug Administration (FDA). The ICPI had grade $3 / 4$ adverse events (AEs) $<10 \%$ lower than Docetaxel (Doc), higher costs and lower adverse events treatment costs (AEsTC). Doc carried a black box waring. Grade 3/4 AEs 3/4 incidence and AEsTC increased beyond 6 cycles. Continued for 12-cycles or one-year, the AEs $3 / 4$ were $>20 \%$ and impairment of quality of life (QoL) was reported. Despite AEs and QoL issues, Doc was considered useful and universally usable in view of low costs and fair value. The OS, grades and value of Doc, Nivo, Atezo and Pembro in "all comers regardless of PDL-1 expression" were limited to < 100 days. Enrichment of PDL1 resulted in unprecedented OS, improved grades and 2-3-foldenhanced value at seemingly justifiable costs.

The present review dealt with many sensitive and controversial subjects, the most notable of which is the continuing debate over 
cost vs. value. The balance between the 2 is sensitive and elusive. Previous attempts to limit drug costs have met with minimal success. The pendulum has recently tipped in favor of value since ASCO and ESMO issued their initiatives in 2013. Costs and value, however, were considered two inseparable sides of one coin (1). Drugs would lose value if patients could not afford the costs. The National Institute for Health and Care Excellence (NICE) in the United Kingdom stipulates that ACER interventions ought not to exceed 20,000-30,000 pounds per quality-adjusted life-year gained. At present, in the US, it is estimated that 1 billion dollars are required to successfully bring a formula from the drawing board to pharmacy shelves. In 2017, the 4-week costs of Nivolumab (Nivo) $240 \mathrm{mg}$ were $\$ 11,914$, Atezolizumab (Atezo) $1200 \mathrm{mg} \$ 11,493$ and Pembrolizumab (Pembro) $200 \mathrm{mg} \$ 11,509$. Costs need to be maintained at certain levels for drug companies to survive, prosper and keep their innovative edge. The US law prohibits discussion of drug costs during the approval process. It is the market, competition and drug assets which decide to favor one drug over another. The costs of either Nivo and Pembro at $10 \mathrm{mg} / \mathrm{Kg}$ would severely curtail use. Combinations of the ICPI with other immune drugs would increase costs and probably the toxicity. Costs of 4 cycles of Ipilimumab (Ipi) at $3 \mathrm{mg} / \mathrm{Kg}$ for a $70 \mathrm{Kg}$ patient were estimated at $\$ 88,200$. Costs of both Nivo and Ipi would limit affordability. In general, large doses, frequent and/ or prolonged administrations would dampen access. High costs could only be tolerated if long-term disease control and/or cure were achieved.

\section{Strategies to improve drug value}

A- In the early clinical studies of the ICPI in NSCLC, various levels of PD-L1 in tumor cells and infiltrating immune cells were used. At present, PD-L1 levels have been defined and expressed with more uniformity. There is $20-30 \%$ response in patients with PD-L1 enhanced expression and $10 \%$ response, some of which were durable, in negative PDL-1. These findings are indicative of the "imperfections" of PD-L1 marker. A panel of markers of PDL1, tumor mutation burden and many others are emerging as more precise and better predictive than PD-L1 mere measurement. From an economic point of view, it would be prudent to spend upfront few hundred dollars on a set of predictive markers than to bear the costs of treating "all comers". This strategy would spare the non- responders the high costs and unnecessary toxicity of ineffective therapy.

B- Research continues to improve the outcome of ICPI in NSCLC $(2,3)$. Combination therapy of Pembro and chemotherapy in locally advanced and metastatic 1-st has significantly improved the OS (KEYNOTE-021). In CHECKMATE 227, Nivolumab Plus Ipilimumab increased the progression-free survival over doublet platinum chemotherapy in chemotherapy-naïve stage IV or recurrent NSCLC. The preliminary OS data were encouraging, and the study will close in 2019.

\section{Conclusions}

Despite Doc AEs gr $3 / 4>20 \%$, negative impact on QoL and a black box warning, the drug was considered useful and universally usable in view of low cost low cost and fair value. The reported OS gains over controls by Doc, Nivo, Atezo and Pembro in all comers in $2^{\text {nd }}$ line NSCLC were significant but modest and limited to $<100$ days. The OS and value by the ICPI in PD-L1 enriched were unprecedented. Further improvement of outcome and value could be achieved using a panel of predictive markers and combination therapy. Successful combinations ought to surmount the hurdles of physical and financial toxicities.

\section{Conflict of interest}

The author has no conflict of interest to declare.

\section{References}

1. Guirgis HM. The impact of PD-L1 on survival and value of the immune check point inhibitors in non-small-cell lung cancer; proposal, policies and perspective. J ImmunoTherapy of Cancer, February 2018. https:// doi.org/10.1186/s40425-018-0320-3

2. Garon EB, Borghaei $\mathrm{H}$ and Stevenson JP. The role of immunotherapy in NSCLC. CME / ABIM MOC Released: 2/27/18.

3. Weiss J, Bauml J, Borghaei H. Locally Advanced Lung Cancer: Is There a New Standard of Care? CME / ABIM MOC Released: 3/27/2018. 